\title{
ON THE DECOMPOSITION OF MODULES
}

\author{
BY ANDREAS DRESS
}

Communicated by Hyman Bass, March 3, 1969

Let $R$ be a commutative ring with $1 \in R, A$ and $R$-algebra-not necessarily commutative - and let $M, N$ be two $A$-left-modules. We write $N-\operatorname{rk}(M) \geqq s$, if $M \cong s N \oplus M^{\prime}$ for some $A$-left-module $M^{\prime}$ with $s \cdot N$ short for $N \oplus N \oplus \cdots \oplus N, s$-times.

Then one can prove the following generalization of a theorem of Serre (cf. [1] or [4]).

THEOREM 1. Assumptions.

(i) $N$ is finitely presented as A-left-module, $\operatorname{End}_{A}(N)$ finitely generated as $R$-module and $M$ a direct summand in a direct sum of finitely presented A-modules;

(ii) the maximal ideal spectrum of $R$ is noetherian of dimensiond;

(iii) for any maximal ideal $\mathfrak{m}$ in $R$ we have $N_{\mathfrak{m}}-\operatorname{rk}\left(M_{\mathfrak{m}}\right) \geqq d+s$ with $N_{\mathfrak{m}}$, resp. $M_{\mathfrak{m}}$ the $A_{\mathfrak{m}}=R_{\mathfrak{m}} \otimes_{R} A$-module $R_{\mathfrak{m}} \otimes_{R} N$, resp. $R_{\mathfrak{m}} \otimes M$.

Then $N-\operatorname{rk}(M) \geqq s$.

Moreover, if $R$ is noetherian, $\hat{R}_{\mathfrak{m}}$ the $\mathfrak{m}$-adic completion of $R$ for some maximal ideal $\mathfrak{m}$ and $\hat{N}_{\mathfrak{m}}$, resp. $\hat{M}_{\mathfrak{m}}$ the $\hat{A}_{\mathfrak{m}}=\hat{R}_{\mathfrak{m}} \otimes_{R} A$-module $\hat{R}_{\mathfrak{m}} \otimes_{R} N$, resp. $\hat{R}_{\mathfrak{m}} \otimes_{R} M$, then

$$
N_{\mathfrak{m}}-\operatorname{rk}\left(M_{\mathfrak{m}}\right) \geqq d+s \Leftrightarrow \hat{N}_{\mathfrak{m}}-\operatorname{rk}\left(\hat{M}_{\mathfrak{m}}\right) \geqq d+s .
$$

One can also prove the following generalization of the Cancellation Theorem of Bass (cf. [1]).

Theorem 2. Assumptions.

(i) and (ii) as in Theorem 1;

(iii) $M$ contains a direct summand $P$ with $N-\mathrm{rk}_{\mathfrak{m}}(P)>d$ for all maximal ideals $\mathrm{m}$ in $R$, which is a direct summand in some $s \cdot N$;

(iv) $Q$ is an A-left-module, which is also a direct summand in some $s \cdot N$, and $M^{\prime}$ is some $A$-left-module with $Q \oplus M \cong Q \oplus M^{\prime}$.

Then $M \cong M^{\prime}$.

The proof follows closely those of Serre and Bass [1], [4], once the following observations have been made:

(1) If $N$ is any $A$-left-module and if $B=\operatorname{End}_{A}(N)$-acting from the right on $N$-then the contravariant functor $\operatorname{Hom}_{A}(\cdot, N)$ from $A$-leftmodules to $B$-right-modules defines a contravariant equivalence between the category $[N]$ of those $A$-left-modules $P$, which are a direct summand in some $s \cdot N$ (and all possible $A$-homomorphisms as morph- 
isms) and the category of finitely generated projective $B$-right-modules. The functor $\operatorname{Hom}_{A}(N, \cdot)$ defines thus an equivalence between $[N]$ and the category of finitely generated projective $B$-left-modules.

(2) If $N$ is a finitely presented $A$-module, $\rho: R \rightarrow \hat{R}$ a ring-homomorphism of $R$ into some commutative ring $\hat{R}$ (with $1 \in \hat{R}$ and $\rho(1)=1)$, such that $\hat{R}$ becomes a flat $R$-module, and if $\hat{M}$, resp. $\hat{N}$ stands for the $\hat{A}=\hat{R} \otimes_{R} A$-module $\hat{R} \otimes_{R} M$, then the natural homomorphism $\hat{R} \otimes_{R} \operatorname{Hom}_{A}(M, N) \rightarrow \operatorname{Hom}_{\hat{A}}(\hat{M}, \hat{N})$ is an isomorphism. (Cf. N. Bourbaki, Algèbre commutative, Chapter 1.)

(3) If $M, N$ are any two $A$-modules and $\phi: M \rightarrow N$ an $A$-homomorphism, define

$$
\begin{aligned}
P(\phi)= & \left\{\phi \psi \in \operatorname{End}_{A}(N) \mid \psi \in \operatorname{Hom}_{A}(M, N)\right\}, \\
I(\phi)= & \left\{\psi \phi \in \operatorname{End}_{A}(M) \mid \psi \in \operatorname{Hom}_{A}(M, N)\right\}, \\
P_{0}(\phi)= & \left\{r \in R \mid r \cdot \operatorname{Id}_{N} \in P(\phi)\right\}, \\
I_{0}(\phi)= & \left\{r \in R \mid r \cdot \operatorname{Id}_{M} \in I(\phi)\right\} \\
& P(\phi) \text { is a right } \operatorname{End}_{A}(N) \text {-ideal, } \\
& I(\phi) \text { is a left } \operatorname{End}_{A}(M) \text {-ideal, } \\
& P_{0}(\phi) \text { and } I_{0}(\phi) \text { are } R \text {-ideals. }
\end{aligned}
$$

The following statements follow easily from (2):

With $\rho: R \rightarrow \hat{R}$ as in (2) and $\hat{\phi}=I d_{\hat{R}} \otimes \phi: \hat{M} \rightarrow \hat{N}$ we have If $N$ is finitely presented, then

$$
P(\hat{\phi})=\widehat{P}(\phi), \quad P_{0}(\hat{\phi})=\widehat{P_{0}(\phi)} .
$$

If $M$ is finitely presented and $N$ a direct summand in a direct sum of finitely presented $A$-modules, then

$$
I(\hat{\phi})=\widehat{I(\phi)}, \quad I_{0}(\hat{\phi})=\widehat{I_{0}(\phi)} .
$$

There are many interesting applications of these observations and the two theorems above. For instance, in the case where $A$ is a separable order over a Dedekind ring $R$, all this specializes to something closely related to the results of Jacobinski [2], [3]. We mention some immediate consequences of (3): If $\rho: R \rightarrow \hat{R}$ is faithfully flat and $N$ finitely presented, then $\phi: M \rightarrow N$ is split-surjective if and only if $\hat{\phi}: \hat{M} \rightarrow \hat{N}$ is split-surjective.

If $N$ is finitely presented, then $\phi: M \rightarrow N$ is split-surjective if and only if $\phi_{\mathfrak{m}}: M_{\mathfrak{m}} \rightarrow N_{\mathfrak{m}}$ is split-surjective for all maximal ideals $\mathfrak{m}$ in $R$.

If $M$ is finitely presented and $N$ a direct summand in a direct sum of finitely presented modules, then $\phi: M \rightarrow N$ is split-injective if and only if all $\phi_{\mathfrak{m}}: M_{\mathfrak{m}} \rightarrow N_{\mathfrak{m}}$ are split-injective. 
It is now not too difficult to go through with the proof of Serre [4], to get Theorem 1, whereas Theorem 2 is now a corollary to Bass's Cancellation Theorem using (1).

\section{REFERENCES}

1. H. Bass, K-theory and stable algebra, Inst. Hautes Etudes Sci. Publ. Math. 22 (1964), 5-60.

2. H. Jacobinski, Über die Geschlechter von Gittern über Ordnungen, J. Reine Angew. Math. 230 (1968), 29-39.

3. - Decomposition of lattices, Acta Math. 121 (1968), 1-29.

4. J-P. Serre, Modules projectifs et espaces fibrés a fibre vectorielle, Séminaire P. Dubreil, M. L. Dubreil-Jacotin et C. Pesot, 1957/58, Fasc. 2, Exposé 23, Secrétariat mathématique, Paris, 1958. 